\title{
AODV Routing Protocol Performance Analysis under MANET Attacks
}

\author{
Mohamed A. Abdelshafy, Peter J. B. King \\ School of Mathematical and Computer \\ Sciences Heriot-Watt University \\ Edinburgh, UK
}

\begin{abstract}
$A O D V$ is a well-known reactive protocol designed for MANET routing. All MANET routing protocols are designed based on the assumption that all nodes cooperate without maliciously disrupting the operation of the routing protocol. In this paper, we study the performance of AODV routing protocol in the presence of some of the well-defined attacks in MANET. We use NS-2 network simulator to analyse the impacts of blackhole, grayhole, selfish and flooding attacks on $A O D V$ protocol performance. While the blackhole and flooding attacks have a severe impact on the AODV performance, the selfish and grayhole attacks have less significant effect on it.
\end{abstract}

\section{Introduction}

Routing protocols in a Mobile Ad Hoc Network (MANET) are designed based on the assumption that all nodes are cooperating to forward data from a source to a destination. So, each node in a MANET acts both as a router and as a host. A large number of routing protocols that are designed to optimize the network performance for MANETs [4] have been developed over the past years. The major issues involved in designing a routing protocol for MANET are node mobility, bandwidth constrained and error prone wireless channel, resource constrained nodes, and dynamic changing of the network topology [1].

MANET routing protocols can be classified as proactive or reactive protocols. In proactive (table-driven) routing protocols, each node maintains one or more tables containing routing information to every other node in the network. While in reactive (on-demand) routing protocols, routes are only created when a source requires to send data to a destination node which means that these protocols are initiated by a source on-demand. In this paper, we concentrate on the AODV protocol [11]. AODV is a reactive protocol, chosen by the IETF for standardization, which has been extensively studied.

Conventional MANET routing protocols assume that all nodes cooperate without maliciously disrupting the operation of the protocol and do not provide defense against malicious attackers [1]. However, the presence of malicious nodes cannot be ignored in computer networks, especially in MANETs because of the wireless nature of the network. MANET inherits security threats that are faced in wired as well as wireless networks and also introduces security attacks unique to itself
[7] due its characteristics. Nodes in MANET have limited computation and power capabilities that make the network more vulnerable to Denial of Service (DoS) attacks. It is difficult to implement cryptography and key management algorithms which need high computations like public key algorithms. Node mobility also introduces a difficulty of distinguishing between stale routes and fake routes. A malicious node can attack the network layer in MANET either by not forwarding packets or by changing some parameters of routing messages such as sequence number and IP addresses, sending fake messages several times and sending fake routing information to disrupt routing operations. There are a large number of existing attacks against MANET [13] and solutions to these attacks. Simulation and study of such attacks [10] [14] allows analysis of the effectiveness defence mechanisms against these types of attacks.

The rest of the paper is organized as follows. In section 2 , an overview of the AODV routing protocol is presented and the impact of some attacks on MANET is discussed. In section 3 , the simulation parameters and results are given. In section 4 concluding remarks are introduced.

\section{AODV Protocol and Security Flaws}

Ad Hoc On-Demand Vector Routing (AODV) [11] is a reactive routing protocol. It uses destination sequence numbers to ensure the freshness of routes and guarantee loop freedom. To find a path to a destination, a node broadcasts a route request (RREQ) packet to its neighbors using a new sequence number. Each node that receives the broadcast sets up a reverse route towards the originator of the RREQ unless it has a fresher one. When the intended destination or an intermediate node that has a fresh route to the destination receives the RREQ, it unicasts a reply by sending a route reply (RREP) packet along the reverse path established at intermediate nodes during the route discovery process. Then the source node starts sending data packets to the destination node through the neighboring node that first responded with an RREP. When an intermediate node along the route moves, its upstream neighbor will notice route breakage due to the movement and propagate a route error (RERR) packet to each of its active upstream neighbors. Routing information is stored only in the source node, the destination node, and the intermediate nodes along the active route which deal with data transmission. This scenario decreases the memory overhead, minimizes the use of network resources, and runs well in high mobility situation. 
The behavior of a malicious node is to disrupt the operation of the AODV routing protocol [7]. The malicious node can spoof source or destination IP address, modify RREQ or RREP packets and/or generate fake RREP or RERR packets. Some of the attacks such as blackhole and grayhole attack are discovered by the source node in connection-oriented protocols such as TCP because the lack of acknowledgments. The source node understands that there is a link error because the destination node does not send ACK packets. If the source node sends out UDP data packets the problem is not detected because UDP is a connectionless protocol.

\subsection{AODV under Flooding Attack}

In a flooding attack [6], a malicious node takes advantage of the route discovery process of the AODV routing protocol. The malicious node aims to flood the network with a large number of RREQs to non-existent destinations in the network which takes a lot of the network resources. Since the destination does not exist in the network, a RREP packet cannot be generated by any node in the network and all the nodes keep on flooding the RREQ packet. When a large number of fake RREQ packets are broadcast into the network, new routes can no longer be added and the network is unable to transmit data packets. Thus, it leads to congestion in the network and overflow of route table in the intermediate nodes so that the nodes cannot receive new RREQ packet, resulting in a DoS attack. Moreover, unnecessary forwarding of these fake RREQ packets has serious effects in MANET [3] as a result of limited computational and power resources of nodes.

However, the AODV protocol can mitigate against this attack by reducing the maximum number of RREQs that a node allowed to send per second.

\subsection{AODV under Selfish Attack}

In MANETs the nodes cooperate to forward data and routing packets from one node to another node. A selfish node is the node that saves its resources; such as battery, by not cooperating in the network operations. A selfish node affects the network performance as it does not correctly process routing or data packets based on the routing protocol. The selfish node behavior is known as a selective existence attack [5]. Selective existence is kind of a passive attack as the node neither participates in the network operation nor changes the content of packets.

The selfish node does not even send any HELLO messages and drops all data and control packets even if these packets are sent to it. When a selfish node needs to send data to another node, it starts working as normal AODV operation. After it finishes sending its data, the node returns to its silent mode and the selfish behavior by dropping all data and routing packets directed through it. Neighbor nodes detect the absence of the selfish node after an interval of silence, and will assume that the node has left their neighborhood. So, they invalidate their own route entries to this node and selfish node becomes invisible to the network.

\subsection{AODV under Grayhole Attack}

In a grayhole attack [8], a malicious node behaves normally as a truthful node during the route discovery process by replying with true RREP messages to the nodes that started RREQ messages. After the source node starts sending data through the malicious node, the malicious node starts dropping these data packets to launch a denial of service (DoS) attack. So, the malicious node forwards routing packets and drops data packets. This selective dropping makes grayhole attacks much more difficult to detect than blackhole attacks. Grayhole attack is also known as node misbehaving attack [2] as the malicious node misleads the network by agreeing to forward the packets in the network.

\subsection{AODV under Blackhole Attack}

In a blackhole attack [12], a malicious node absorbs the network traffic and drops all packets. To carry out a blackhole attack, a malicious node waits for incoming RREQ packets from other nodes. When the malicious node receives an RREQ message, without checking its routing table, it immediately sends a false RREP with a high sequence number and zero hop count to spoof its neighbours that it has the best route to the destination. Thus, the malicious node reply will be received by the source node before any reply from other nodes. When a source node receives multiple RREP, it chooses the RREP with the largest destination sequence number and the smallest hop count. Then the source node ignores other RREP packets and begins sending data packets over the malicious node. When the data packets routed by the source node reach the blackhole node, it drops the packets rather than forwarding them to the destination node.

The malicious node attacks all RREQ packets in this way and takes over all routes. Therefore all packets are sent to a point where they are not forwarding anywhere. If the malicious node generates false RREP messages that appear to come from another victim node, all messages will be forwarded to the victim node. By doing this, victim node will have to process all incoming messages and is subjected to a sleep deprivation attack.

\section{Attack Simulations}

We simulated various attacks on the AODV protocol using the ns-2 simulator [9]. The parameters used are shown in Table I. Node mobility was modelled with the random waypoint method. Our simulation results is obtained from 3 different movement scenarios, 3 different traffic scenarios and 3 different node-type (malicious or non-malicious) scenarios which means that each metric value is the mean of the 27 runs. In all cases, the $90 \%$ confidence interval was small compared with the values being reported. While we examined the effects of the attacks on both UDP and TCP traffic, in this paper we focused on their impact on the TCP traffic only. We also examined the effect of these attacks for different node speeds $(0,5,10,15$, $20,25$ and $30 \mathrm{~m} / \mathrm{s})$. The paper results are focused on the static network $(0 \mathrm{~m} / \mathrm{s})$ and the high mobility network $(30 \mathrm{~m} / \mathrm{s})$.

Packet Delivery Ratio (PDR): This is the ratio of packets that are successfully delivered to a destination compared to 
TABLE I. Simulation Parameters

Simulation Time

Simulation Area

Number of Nodes

Number of Connections

Number of Malicious Nodes

Node Speed

Pause Time

Traffic Type

the number of packets that have been sent out by the sender. Throughput: This is the number of data bits delivered to the application layer of destination node in unit time measured in bps.

End-to-End Delay (EED): This is the average time taken for a packet to be transmitted across the network from source to destination.

Routing Overhead: This is the number of routing packets for route discovery and route maintenance needed to send to deliver the data packets from sources to destinations.

Normalized Routing Load (NRL): This is the total number of routing packets transmitted by the number of received data packets.

Route Discovery Latency (RDL): This is the average delay between the sending RREQ from a source and receiving the first corresponding RREP.

Sent Data Packets: This is the total number of packets sent by all source nodes during the simulation time.

\subsection{AODV under Flooding Attack}

Figure 1 shows the effect of malicious nodes on the packet delivery ratio for static nodes and for high node mobility. The result shows that the packet delivery ratio decreases while increasing the number of malicious nodes in the network and this decrease is independent of the node mobility. The graph shows that while the PDR decreases by $3 \%$ if there are 5 malicious nodes for static nodes, the PDR decreases by $5.5 \%$ for the same number of malicious nodes in high node mobility.

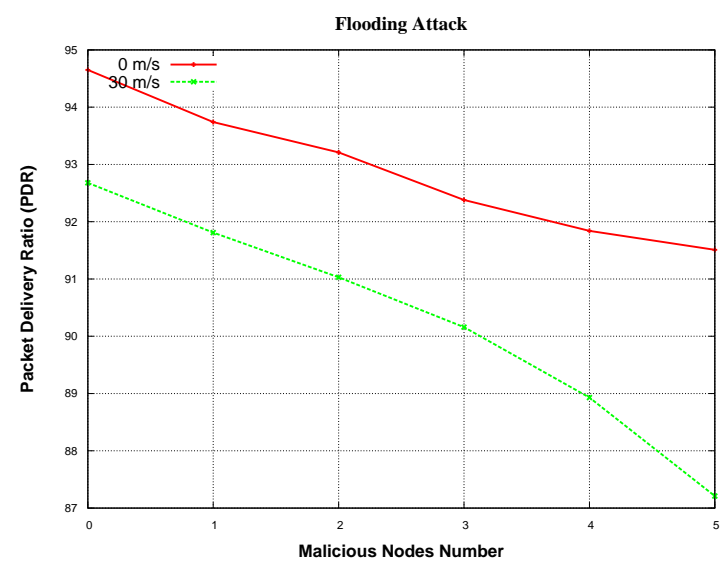

Figure 1. PDR under Flooding Attack

Figure 2 shows the effect of malicious nodes on the network throughput for static nodes and for high node mobility. The result shows that the throughput decreases by $10 \%$ for each malicious node introduced in the network and this decrease is independent of the node mobility.

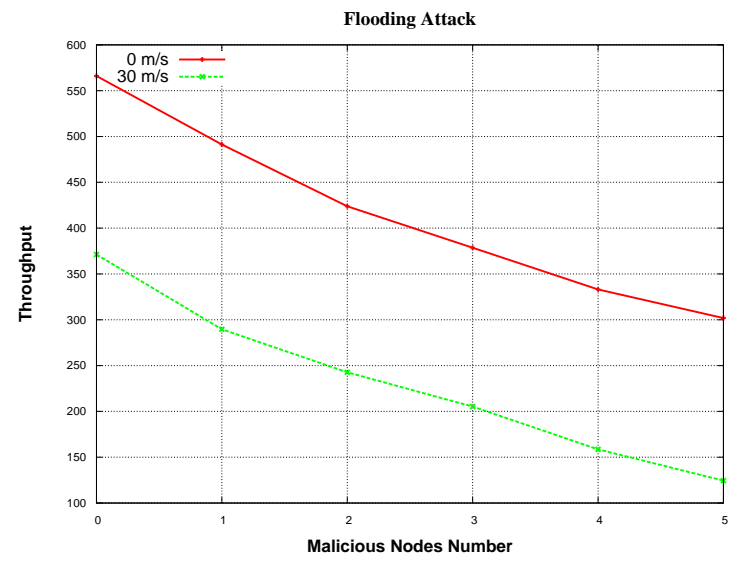

Figure 2. Throughput under Flooding Attack

The effect of malicious nodes on the end-end-delay for static nodes and for high node mobility is shown in Figure 3. The result shows that the delay has no significant change for the first malicious node while the delay increases as more malicious nodes are added in the network up to $30 \%$ in the average if there are 5 malicious nodes and this increase is independent of the node speed.

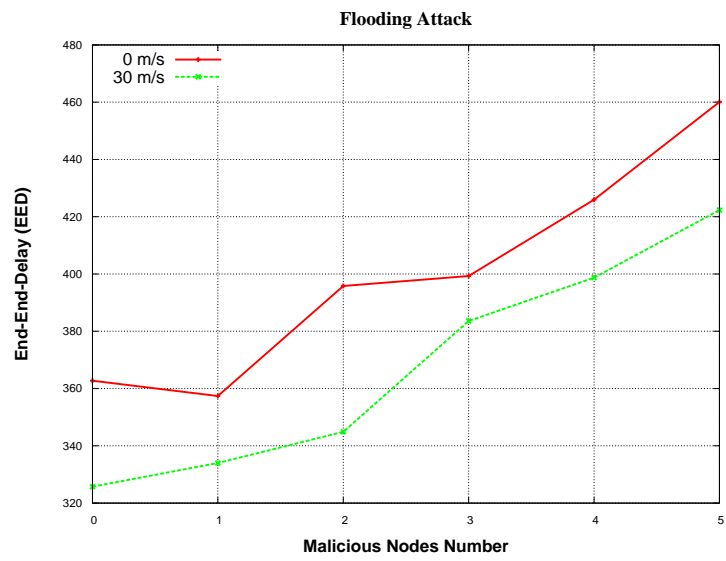

Figure 3. EED under Flooding Attack

Figure 4 shows the effect of malicious nodes on the routing overhead for static nodes and for high node mobility. The result shows that the routing overhead linearly increases by $50 \%$ in the average as the number of malicious nodes in the network increases for static nodes while the increase is about $70 \%$ for high node mobility.

The effect of malicious nodes on the normalized routing load for static nodes and for high node mobility is shown in Figure 5. For static network, NRL increases linearly by $80 \%$ for each added malicious node. With high mobile nodes, the increase is about $100 \%$ for each added malicious node, 


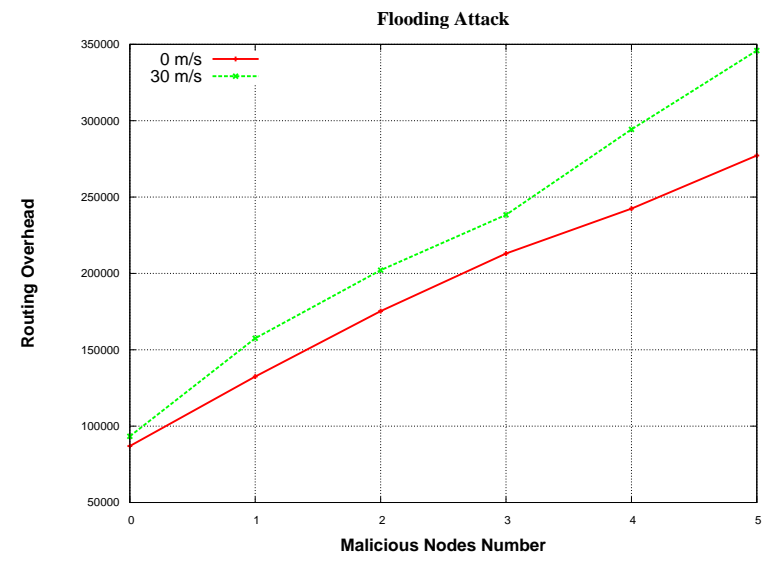

Figure 4. Routing Overhead under Flooding Attack

although the role of increase appears to be greater for large number of malicious nodes.

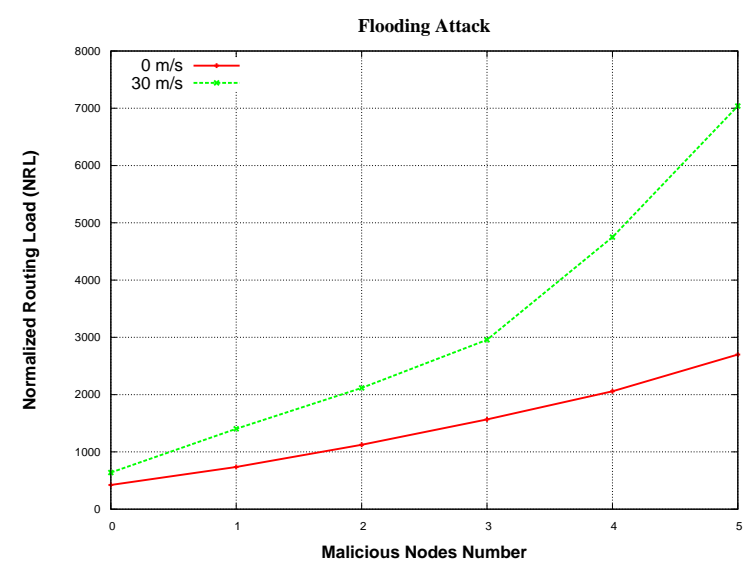

Figure 5. NRL under Flooding Attack

The effect of malicious nodes on the routing discovery latency for static nodes and for high node mobility is shown in Figure 6. The result shows that RDL increases for each malicious node introduced in the network and this increase is independent of the node mobility. For small number of malicious nodes, the RDL is less for a mobile network, but as the number of malicious nodes increases, the highly mobile network will display a higher RDL than a static network.

Figure 7 shows the effect of malicious nodes on the total number of packets sent by all sources for static nodes and for high node mobility. The result shows that the total number of data packets sent by all the source nodes decreases by $10 \%$ for each malicious node introduced in the network and this decrease is independent of the node mobility.

\subsection{AODV under Selfish Attack}

Figure 8 shows the effect of malicious nodes on the packet delivery ratio for static nodes and for high node mobility. The result shows that the packet delivery ratio has no significant

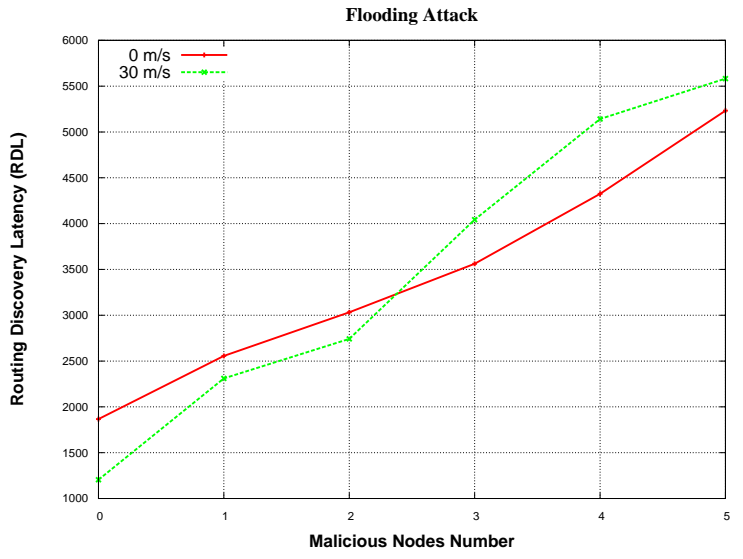

Figure 6. RDL under Flooding Attack

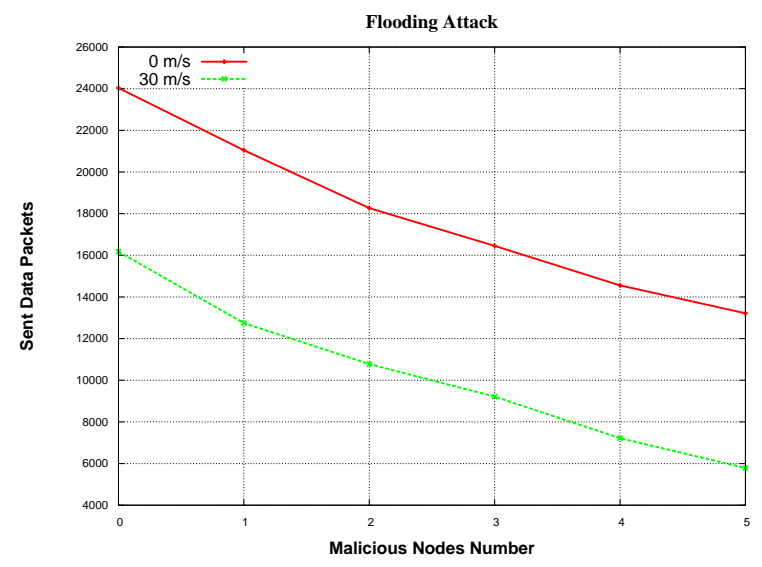

Figure 7. Sent Data under Flooding Attack

change as the number of malicious nodes in increased in the network and this is independent of the node mobility.

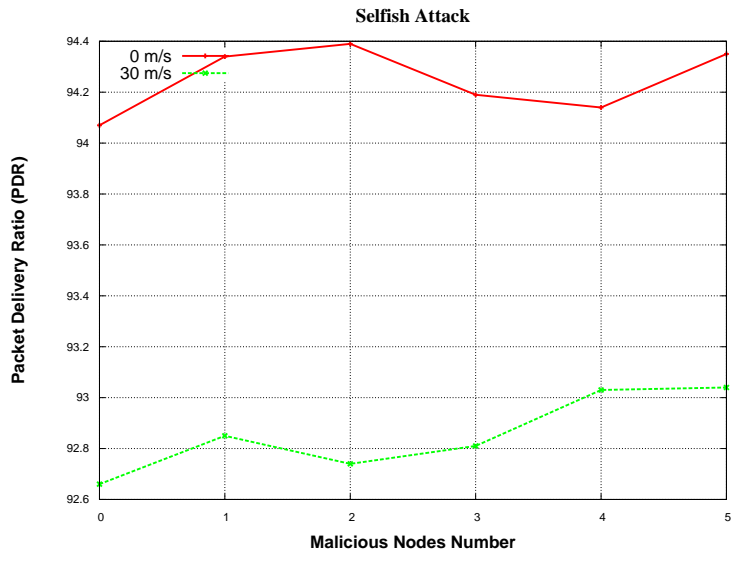

Figure 8. PDR under Selfish Attack

Figure 9 shows the effect of malicious nodes on the network throughput for static nodes and for high node mobility. The result shows that while the throughput of high mobility 
nodes decreases by $40 \%$ relative to the static nodes, the throughput does not affected by the number of malicious nodes in the network.

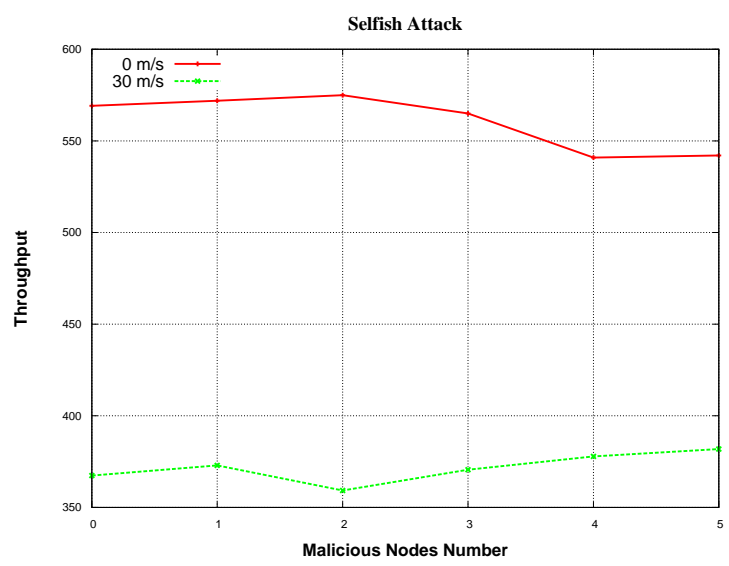

Figure 9. Throughput under Selfish Attack

The effect of malicious nodes on the end-end-delay for static nodes and for high node mobility is shown in Figure 10. The result shows that the delay has no significant change as the number of malicious nodes in increased in the network and this is independent of the node mobility.

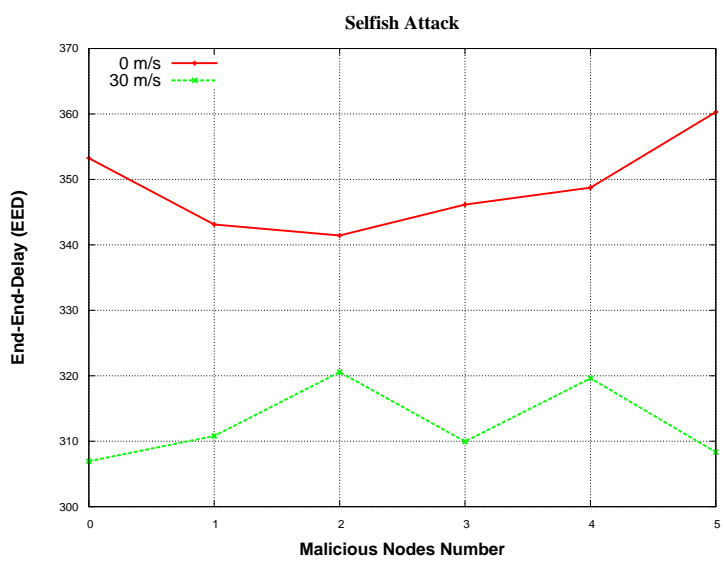

Figure 10. EED under Selfish Attack

Figure 11 shows the routing overhead under the selfish attack, as the number of malicious nodes increases. For the static network, the first two malicious nodes have little effect; and adding future malicious nodes reduces the overhead slightly. In the highly mobile network, there is a significant reduction in overhead for each malicious node added.

These results has slightly confusion as the selfish attack improves the routing overhead. The explanation is that the routing overhead is only measured on the number of routing packets which decreases as the result of increasing the malicious node number because dropping of these packets by these malicious nodes.

The effect of malicious nodes on the normalized routing load for static nodes and for high node mobility is shown in

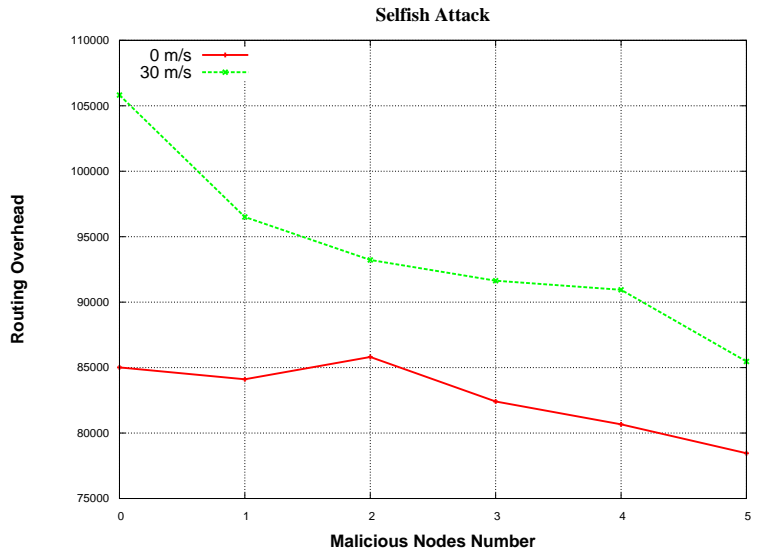

Figure 11. Routing Overhead under Selfish Attack

Figure 12. The result shows that while NRL has no significant change in the case of static nodes, it decreases by $5 \%$ on the average for each malicious node in the case of high mobility nodes. Because NRL as well is measured on the number of routing packets which decreases as the result of increasing the malicious node number, NRL is slightly improved as increasing the number of malicious nodes.

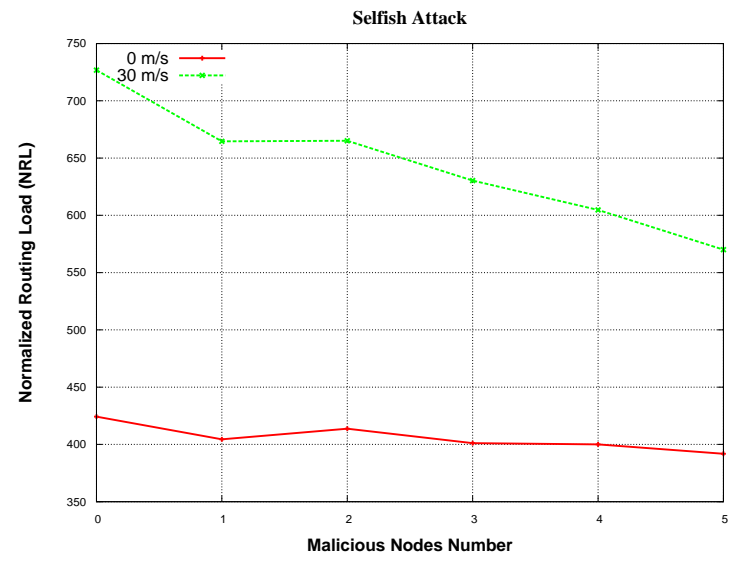

Figure 12. NRL under Selfish Attack

The effect of malicious nodes on the routing discovery latency for static nodes and for high node mobility is shown in Figure 13. The result shows that RDL for the high mobility nodes is better that the static nodes by $25 \%$ on the average whatever the number of malicious nodes in the network.

Figure 14 shows the effect of malicious nodes on the total number of packets sent by all sources for static nodes and for high node mobility. The result shows that the total number of data packets sent by all the source nodes has not significant change as a results of malicious nodes presence in the network and this is independent of the node mobility.

\subsection{AODV under Grayhole Attack}

As the grayhole node drops all data packets and the selfish node drops all data and routing packets, the grayhole 


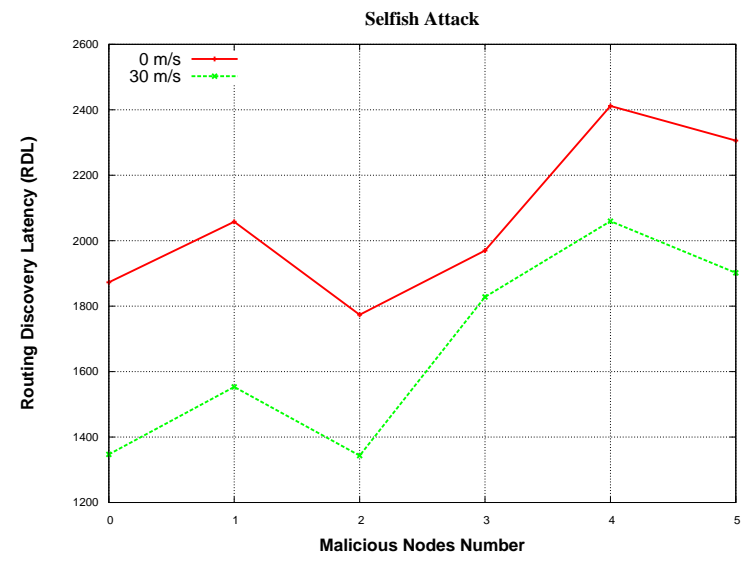

Figure 13. RDL under Selfish Attack

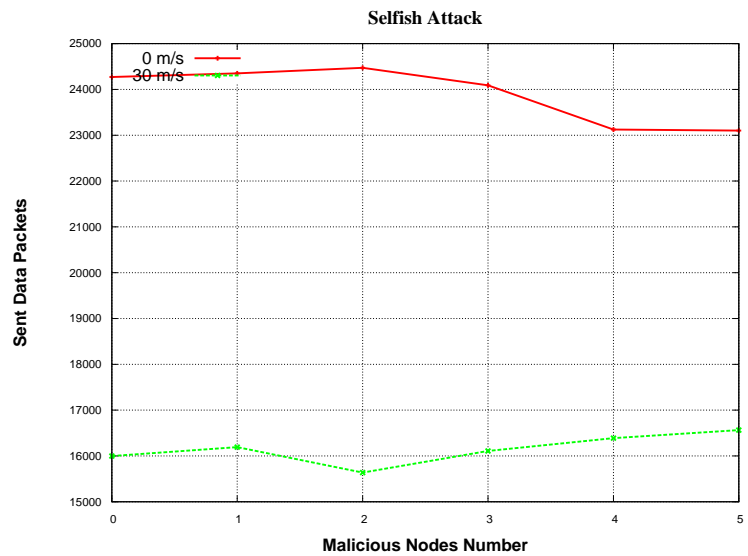

Figure 14. Sent Data under Selfish Attack

attack simulation produces very similar results to the selfish attack. This is because of the packet delivery ratio, network throughput, end-end-delay, normalized routing load and routing overheads are calculated based on the received data packets which are the identical for the same simulation scenario.

Figure 15 shows the effect of malicious nodes on the packet delivery ratio for static nodes and for high node mobility. The result shows that the packet delivery ratio does not change significantly as the number of malicious nodes in increased in the network and this is independent of the node mobility.

Figure 16 shows the effect of malicious nodes on the network throughput for static nodes and for high node mobility. The result shows that while the throughput of high mobility nodes decreases by $35 \%$ relative to the static nodes, the throughput is not affected by the number of malicious nodes in the network.

The effect of malicious nodes on the end-end-delay for static nodes and for high node mobility is shown in Figure 17. The result shows that the delay of high mobility nodes better by $10 \%$ relative to the static nodes and the delay does not affected by the number of malicious nodes in the network.

Figure 18 shows the effect of malicious nodes on the routing overhead for static nodes and for high node mobility.

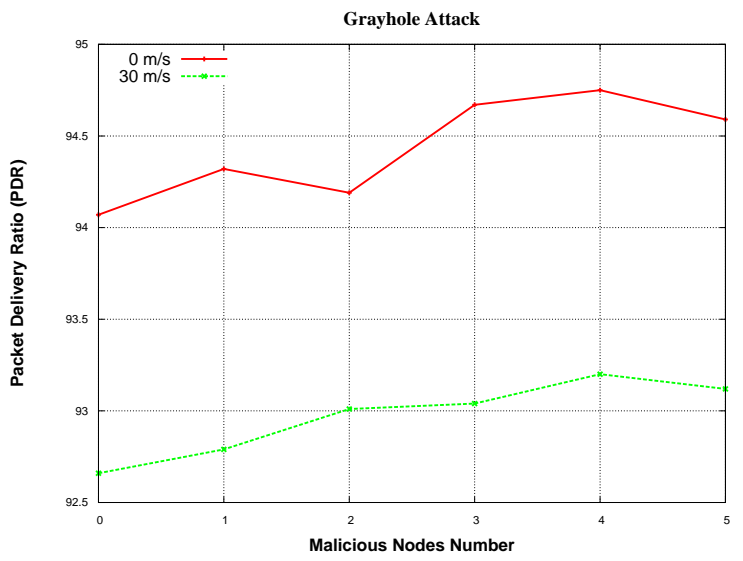

Figure 15. PDR under Grayhole Attack

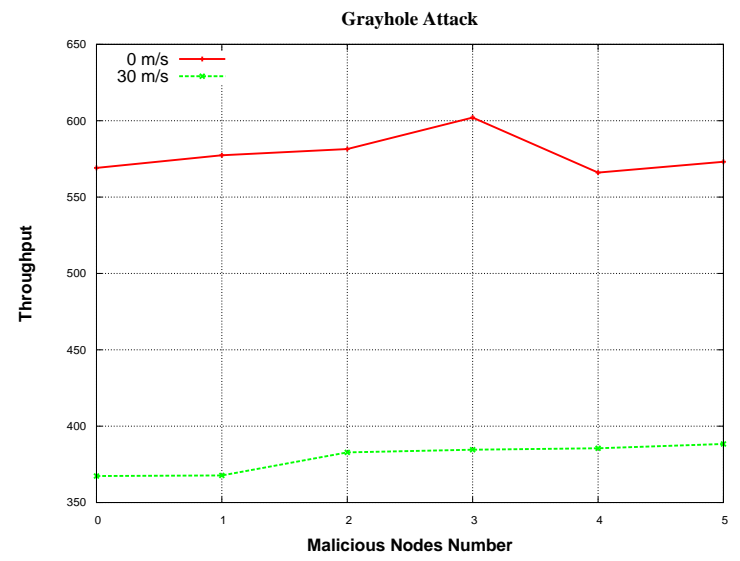

Figure 16. Throughput under Grayhole Attack

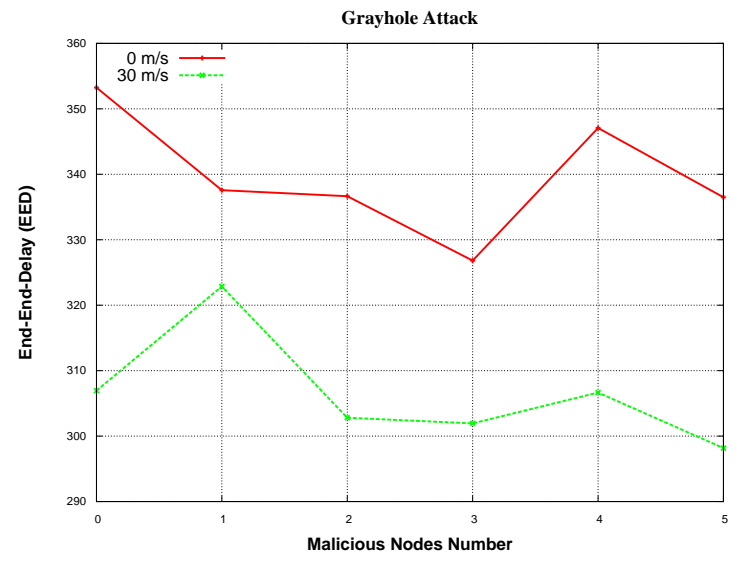

Figure 17. EED under Grayhole Attack

The result shows that the routing overhead for static nodes is better by about $10 \%$ than its value in high mobility nodes and while it decreases by $5 \%$ for each malicious node in the case of high mobility nodes, the routing overhead decreases by $3 \%$ for each malicious node in the case of static nodes.

The effect of malicious nodes on the normalized routing 


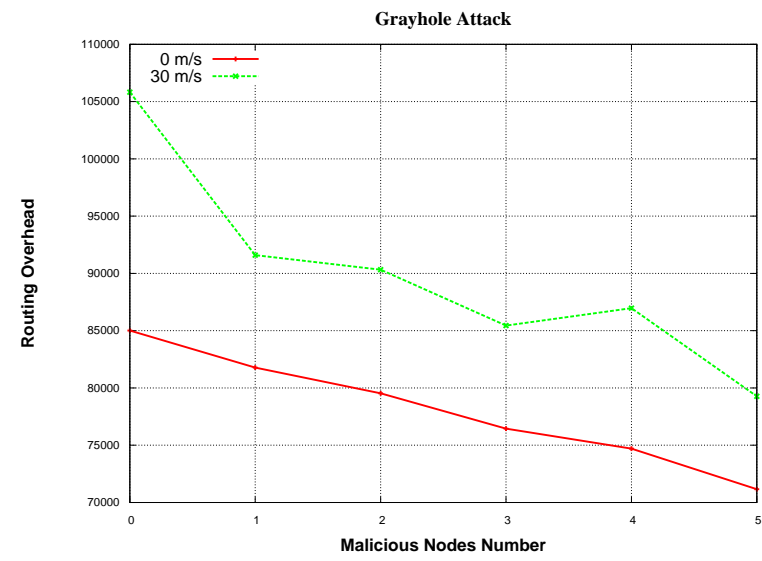

Figure 18. Routing Overhead under Grayhole Attack

load for static nodes and for high node mobility is shown in Figure 19. The result shows that while NRL for static nodes is better by about $40 \%$ than its value in high mobility nodes and while it decreases by $6 \%$ for each malicious node in the case of high mobility nodes, the routing overhead decreases by $4 \%$ for each malicious node in the case of static nodes.

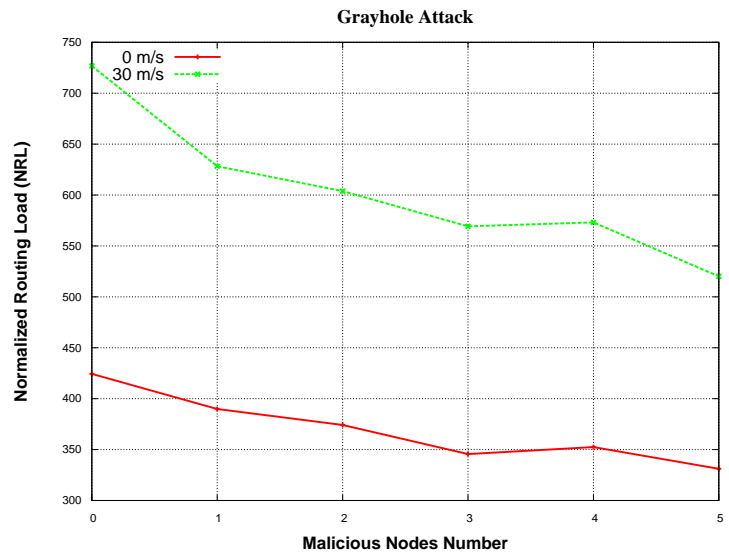

Figure 19. NRL under Grayhole Attack

The effect of malicious nodes on the routing discovery latency for static nodes and for high node mobility is shown in Figure 20. The result shows that RDL for the high mobility nodes is better that the static nodes by $20 \%$ on the average whatever the number of malicious nodes in the network.

Figure 21 shows the effect of malicious nodes on the total number of packets sent by all sources for static nodes and for high node mobility. The result shows that the total number of data packets sent by all the source nodes has no significant change as result of malicious nodes in the network and this is independent of the node mobility.

\subsection{AODV under Blackhole Attack}

Figure 22 shows the effect of malicious nodes on the packet delivery ratio for static nodes and for high node mobility. The

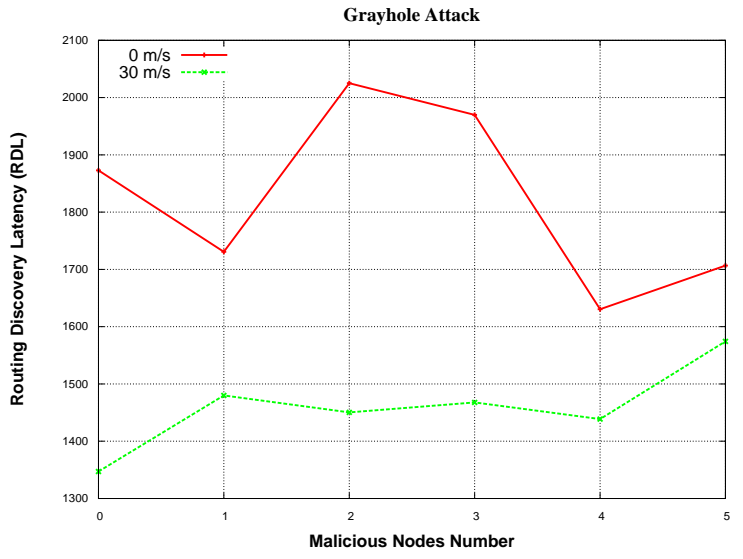

Figure 20. RDL under Grayhole Attack

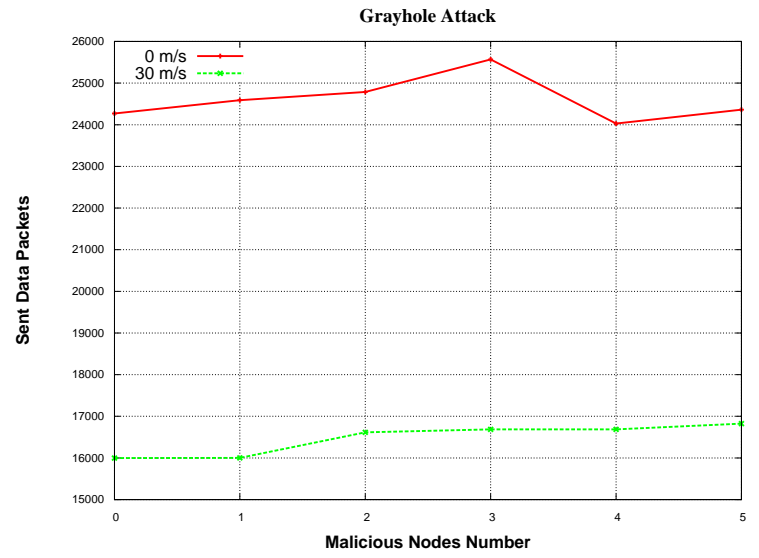

Figure 21. Sent Data under Grayhole Attack

result shows that the packet delivery ratio has no significant change for the first malicious node while the packet delivery ratio decreases by about $6 \%$ for each malicious node in the network and this decrease is independent of the node mobility.

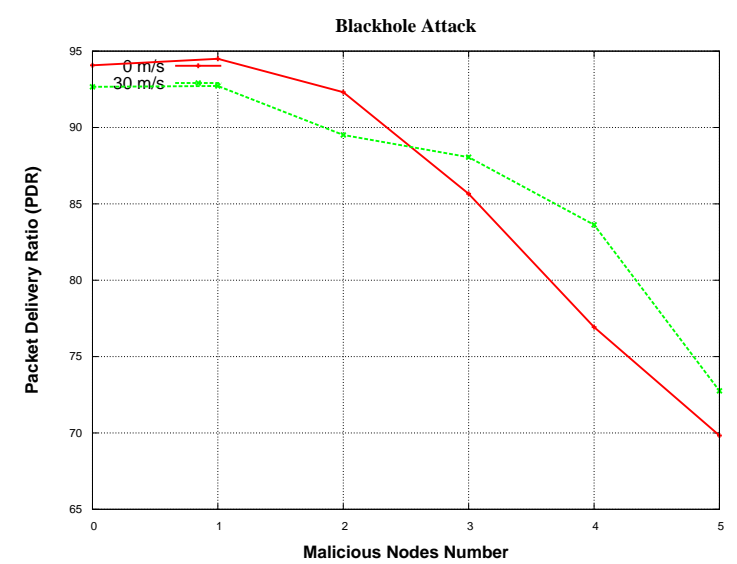

Figure 22. PDR under Blackhole Attack 
Figure 23 shows the effect of malicious nodes on the network throughput for static nodes and for high node mobility. The result shows that the throughput for the static nodes is better that the high mobility nodes by $15 \%$ and the throughput decreases by $20 \%$ for each malicious node introduced in the network and this decrease is independent of the node mobility.

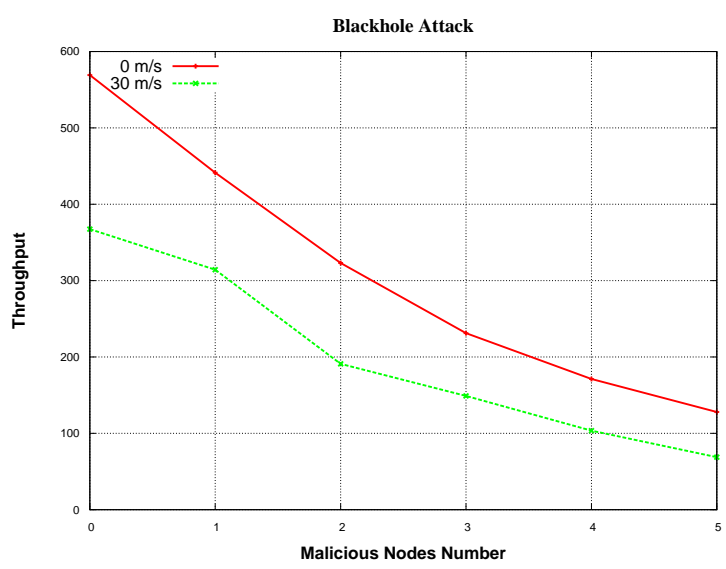

Figure 23. Throughput under Blackhole Attack

The effect of blackhole attack on the end-end-delay is shown in Figure 24. The first two malicious nodes reduce the delay significantly; independently of whether the network is static or highly mobile. Subsequent malicious nodes decrease the delay further.

These results are slightly paradoxical as the attack improves the delay. The explanation is that the delay is only measured on packets that reach their destinations. Other packets that would have reduced the throughput are disappearing into blackhole nodes, so the routes that avoid blackhole nodes suffer less competition.

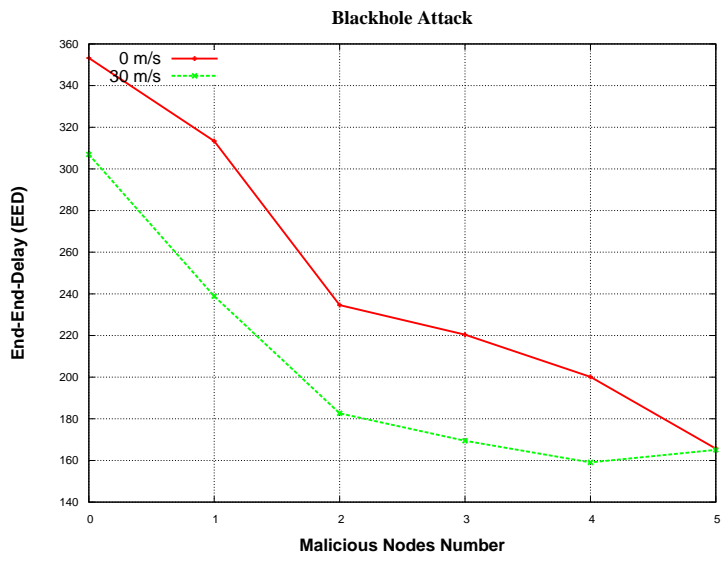

Figure 24. EED under Blackhole Attack

Figure 25 shows the effect of malicious nodes on the routing overhead for static nodes and for high node mobility. The result shows that the routing overhead has not significant change as a result of malicious nodes presence in the network and this is independent of the node mobility. These results have slightly confusion as the blackhole attack improves the routing overhead. This is because the blackhole nodes stop rebroadcasting the RREQ which decreases the RREQ packets number that is one of factors used to measure the routing overhead.

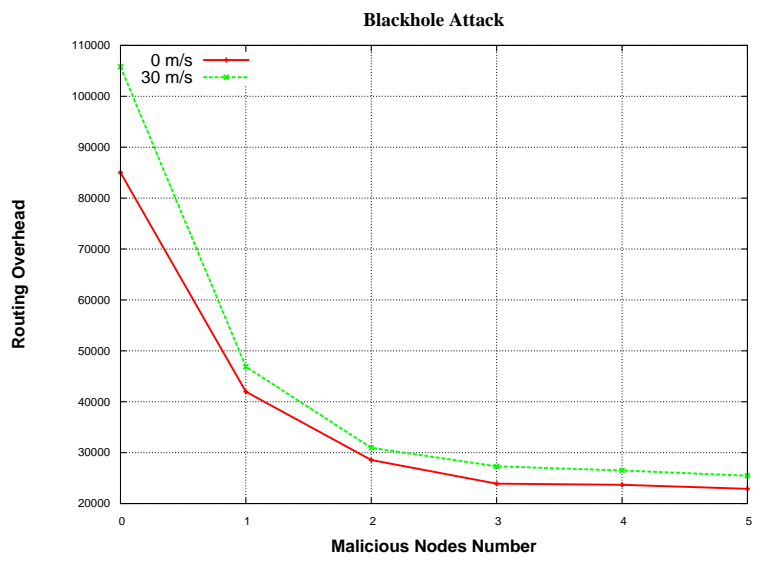

Figure 25. Routing Overhead under Blackhole Attack

The effect of malicious nodes on the normalized routing load for static nodes and for high node mobility is shown in Figure 26. The result shows that the first three malicious nodes have very little impact on the NRL while the presence of other malicious nodes dramatically increases NRL. While this conclusion is true for both mobile and static node, the effect on static node is more remarkable.

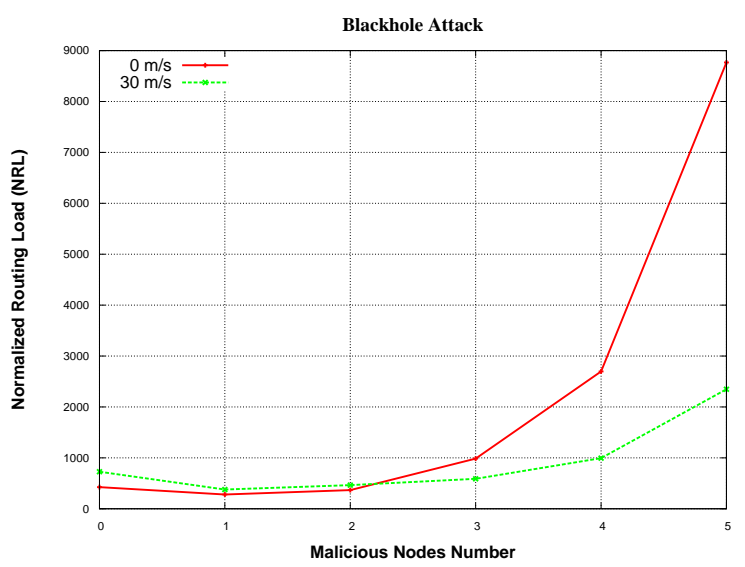

Figure 26. NRL under Blackhole Attack

The effect of malicious nodes on the routing discovery latency for static nodes and for high node mobility is shown in Figure 27. While the result shows that RDL decreases dramatically for each malicious node introduced in the network which is a positive sign, this is a fake advantage because the RDL is computed based on the difference between RREQ and RREP times. Since a malicious node under blackhole attack replies with a fake RREP, RDL becomes smaller under attack. 


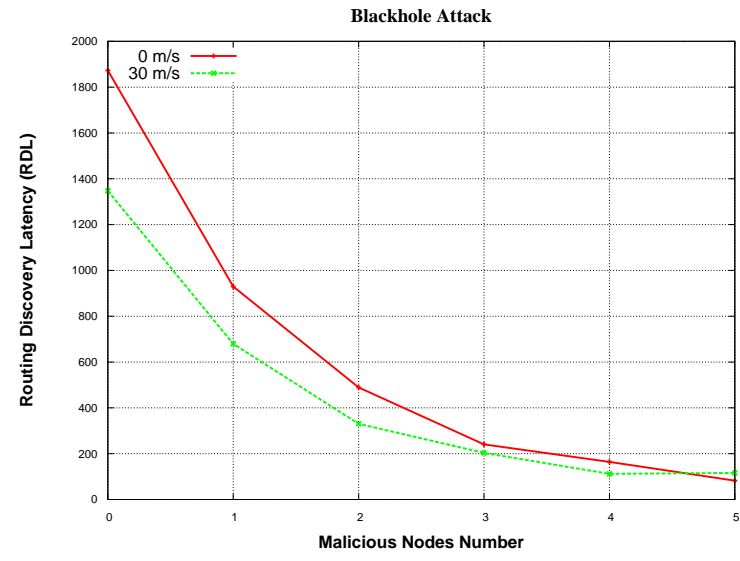

Figure 27. RDL under Blackhole Attack

Figure 28 shows the effect of malicious nodes on the total number of packets sent by all sources for static nodes and for high node mobility. The result shows that the total number of data packets sent by all the source nodes increases in static nodes by $30 \%$ than in high mobility nodes and these packets decreases by $15 \%$ for each malicious node introduced in the network and this decrease is independent of the node mobility.

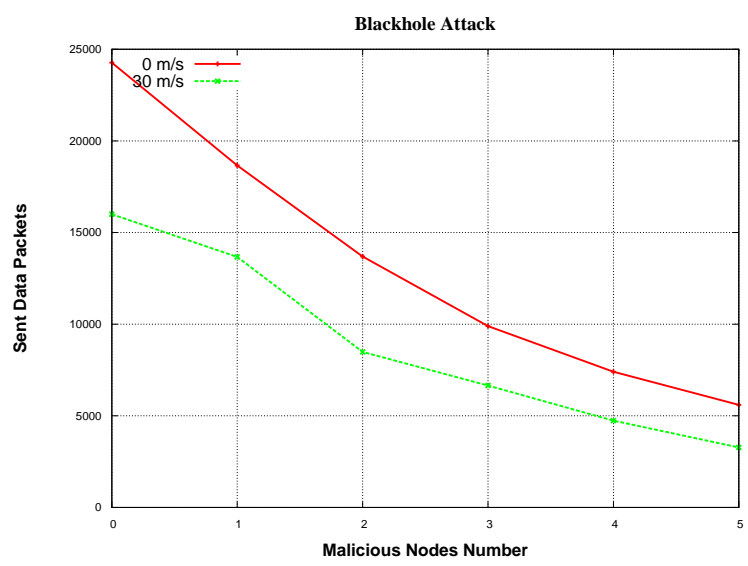

Figure 28. Sent Data under Blackhole Attack

\section{Conclusion}

In this paper, we analyse the impact of some of the attacks on the AODV routing protocol. The flooding, selfish, grayhole and blackhole attacks are simulated using NS-2 network simulator to study their effects on the performance metrics such as packet delivery ratio, network throughput, end-end-delay, routing overhead, normalized routing load, routing discovery latency and sent data packets.

From the simulation, we conclude that the blackhole and flooding attacks have dramatic impact on all the network metrics while the selfish and grayhole attacks do not affect so much in these metrics because both attacks drop the data packets which are the major factor in calculating these metrics. While selfish and grayhole attack share the data dropping, the blackhole introduce a fake RREP which affects the network performance and the flooding attack introduces a fake RREQ which affects the network performance as well.

\section{References}

[1] M. Abdelshafy and P. J. B. King. Analysis of security attacks on aodv routing. In 8th International Conference for Internet Technology and Secured Transactions (ICITST-2013), pages 1-6, London, UK, 2013.

[2] M. Arya and Y. K. Jain. Grayhole attack and prevention in mobile adhoc network. International Journal of Computer Applications, 27(10):2126, August 2011.

[3] A. Bandyopadhyay, S. Vuppala, and P. Choudhury. A simulation analysis of flooding attack in MANET using ns-3. In Wireless Communication, Vehicular Technology, Information Theory and Aerospace Electronic Systems Technology (Wireless VITAE), 2011 2nd International Conference on, pages 1-5, 2011.

[4] A. Boukerche, B. Turgut, N. Aydin, M. Ahmad, L. Bölöni, and D. Turgut. Routing protocols in ad hoc networks: a survey. Computer Networks, 55(13):3032-3080, September 2011.

[5] P. Goyal, S. Batra, and A. Singh. A literature review of security attack in mobile ad-hoc networks. International Journal of Computer Applications, 9(12):11-15, November 2010.

[6] Y. Guo and S. Perreau. Detect DDoS flooding attacks in mobile ad hoc networks. Int. J. Secur. Netw., 5(4):259-269, Dec. 2010.

[7] A. Kumar. Security attacks in MANET - a review. IJCA Proceedings on National Workshop-Cum-Conference on Recent Trends in Mathematics and Computing 2011, RTMC(11), May 2012.

[8] K. Manikandan, R.Satyaprasad, and K.Rajasekhararao. A Survey on Attacks and Defense Metrics of Routing Mechanism in Mobile Ad hoc Networks. IJACSA - International Journal of Advanced Computer Science and Applications, 2(3):7-12, 2011.

[9] The network simulator ns-2. http://www.isi.edu/nsnam/ns/.

[10] M. Patel and S. Sharma. Detection of malicious attack in manet a behavioral approach. In Advance Computing Conference (IACC), 2013 IEEE 3rd International, pages 388-393, 2013.

[11] C. E. Perkins and E. M. Royer. Ad-hoc on-demand distance vector routing. In Proceedings of the 2nd IEEE Workshop on Mobile Computing Systems and Applications, pages 90-100, 1997.

[12] N. Sharma and A. Sharma. The black-hole node attack in MANET. In Proceedings of the 2012 Second International Conference on Advanced Computing \& Communication Technologies, ACCT '12, pages 546-550, Washington, DC, USA, 2012. IEEE Computer Society.

[13] M. Singh, A. Singh, R. Tanwar, and R. Chauhan. Security attacks in mobile adhoc networks. IJCA Proceedings on National Workshop-CumConference on Recent Trends in Mathematics and Computing 2011, RTMC(11), May 2012.

[14] G. Usha and S. Bose. Impact of gray hole attack on adhoc networks. In Information Communication and Embedded Systems (ICICES), 2013 International Conference on, pages 404-409, 2013. 